\section{Th Microwave Engineering Ulm University}

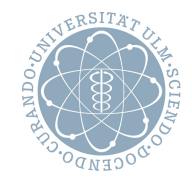

ulm university universität

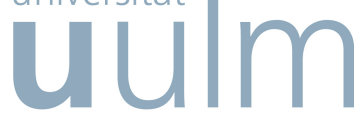

\title{
Substrate Integrated Waveguide Slot-Fed Grid Array Antenna
}

\author{
Osama Khan, Johannes Meyer, Klaus Baur, and Christian Waldschmidt
}

(C) 2015 IEEE. Personal use of this material is permitted. Permission from IEEE must be obtained for all other uses, in any current or future media, including reprinting/republishing this material for advertising or promotional purposes, creating new collective works, for resale or redistribution to servers or lists, or reuse of any copyrighted component of this work in other works. 


\title{
Substrate Integrated Waveguide Slot-Fed Grid Array Antenna
}

\author{
Osama Khan ${ }^{1}$, Johannes Meyer ${ }^{1}$, Klaus Baur ${ }^{1}$, and Christian Waldschmidt ${ }^{2}$ \\ ${ }^{1}$ Engineering Components Radar \\ Robert Bosch GmbH, Leonberg, Germany \\ Email: osama.khan@de.bosch.com \\ ${ }^{2}$ Institute of Microwave Engineering \\ University of Ulm, Ulm, Germany \\ Email: christian.waldschmidt@uni-ulm.de
}

\begin{abstract}
A planar substrate integrated waveguide slot-fed microstrip grid array antenna for the frequency range $76-81 \mathrm{GHz}$ is presented. The antenna is realized on a standard soft RF substrate. It is fed using a transverse slot in a substrate integrated waveguide on a separate substrate layer below the antenna. A wideband microstrip to substrate integrated waveguide is designed to feed the antenna. Measurement results show a $12 \%$ S11<-10 dB bandwidth.
\end{abstract}

Index Terms-Grid array, millimeter-wave antennas, microstrip antennas, substrate integrated waveguide

\section{INTRODUCTION}

In recent years, there has been a rise in demand in commercial applications using millimeter-wave frequencies. Highly directional radio links as well as radar sensors are examples of applications which make use of these frequencies. The frequency range $76-81 \mathrm{GHz}$ has been allocated to automotive radar applications [1]. There is great interest in designing low cost and high performance sensors for these applications.

An important component of a radar sensor is the antenna. Single layer planar and cost effective antenna concepts that allow easy integration in the sensor, such as the microstrip patch array or slotted substrate integrated waveguide (SIW) antennas suffer from narrow impedance bandwidths [2]. Additionally, as these linear antenna arrays are conventionally fed from one side, their radiation pattern stability bandwidths are also narrow. The grid antenna array has received a lot of attention recently as an alternative for millimeter-wave applications.

After being reported by Krauss [3] who introduced it as a backward angle-fire wire antenna, the grid array antenna has been realized in microstrip technology [4]. Recently, low temperature co-fired ceramics (LTCC) technology has been used to realize multi-layer feeding networks [5], [6]. All these microstrip concepts involve a via feed for the antenna. Due to impedance matching requirements, these vias are generally required to be extremely thin. A single layer linear array using a differential feed was realized on a soft substrate using a waveguide to microstrip transition [7].

This paper presents a multilayer microstrip grid array antenna designed on a soft RF substrate. The novelty of this paper is the introduction of a simple transverse slot feed for the antenna array. The slot enables a phase difference of $180^{\circ}$ between the corresponding elements without any additional structures, which enables a symmetric single feed array design. This paper is organized as follows: Section II describes briefly the antenna array and the feed design. First, the SIW based feed is presented, followed by the array design. A microstrip to SIW transition is also described as part of the antenna feeding network. Section III provides simulation and measurement results. Finally, the conclusion is provided in Section IV.

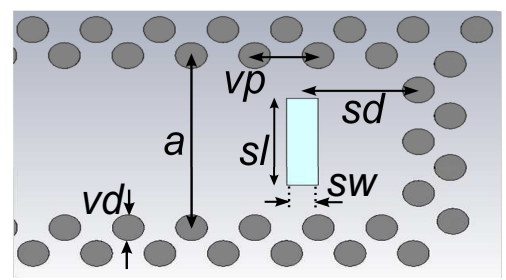

Figure 1: SIW with transverse slot

\section{DESIGN PROCEDURE}

The grid array is designed on a low loss RF substrate layer ( $h=0.127 \mathrm{~mm}, \epsilon_{r}=3.1$ ), backed by a low-loss prepreg of thickness $0.127 \mathrm{~mm}$. The RF substrate layer is partially metalized to include the microstrip to SIW transition. Below the prepreg is the SIW, also realized on the same RF substrate material as the top layer with thickness $0.127 \mathrm{~mm}$ for the SIW feed. The bottom substrate is completely metalized on both sides, so that the top metalization functions as the ground plane for the antenna. Thus, the antenna has an effective substrate thickness of $0.254 \mathrm{~mm}$.

\section{A. SIW transverse slot feed}

With regards to the mechanical construction of the PCB, a slot feed removes the need for thin vias for the antenna feed, which are difficult to realize. An additional benefit of a transverse slot feed is that a $180^{\circ}$ phase difference between the relevant electric field components in the array is achieved without any additional structures. In comparison, for previous realizations, this phase difference between two feed points was realized through different methods, such as asymmetric power 
dividers [6], rat race power dividers [8], microstrip differential feeding [7] or other similar structures.

An SIW feed is designed, consisting of a shorted SIW with a transverse slot to couple the energy to the antenna. It is shown in Fig. 1. The slot couples the energy to the top layer where the antenna is located. Important design parameters for the SIW feed are the SIW width $a$, the slot length $s l$, and the distance between the slot and the via wall short $s d$. A similar feeding method has been used as a wideband coupling method for millimeter-wave microstrip patch antennas [9]. The SIW consists of a substrate with top and bottom copper metalization of thickness $18 \mu \mathrm{m}$. The walls of the SIW consist of vias with diameter $v d$ and spacing $v p$ of $300 \mu \mathrm{m}$ and $600 \mu \mathrm{m}$ respectively. The SIW width, measured between via centers is chosen to be $a=2.0 \mathrm{~mm}$. With the SIW height being the substrate height of $0.127 \mathrm{~mm}$, this ensures that the only propagating mode in the operating frequency range of 76$81 \mathrm{GHz}$ is the $\mathrm{TE}_{10}$ mode. A transverse slot is etched on the upper metalization of the SIW, which also functions as the ground plane of the antenna. The slot length $s l$ and distance to the via wall $s d$ are chosen to be around $\lambda_{g} / 2$ to achieve slot resonance, where $\lambda_{g}=\lambda_{0} / \sqrt{\epsilon_{r}}$ is the wavelength in the SIW at the operating frequency.

Full wave simulations with the T-Solver of CST Microwave Studio are performed. In the first simulation step, the slot is coupled to a microstrip line on the top substrate, terminated on both sides with waveguide ports. It is observed that the slot couples to the microstrip line over a wide frequency band without radiating into free space. This is the desired behavior, as it allows independent design of the antenna array and feed network.

\section{B. Array design}

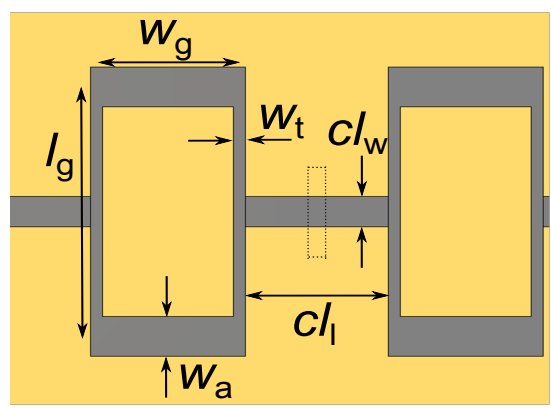

Figure 2: Grid antenna element

Afterwards, a grid antenna array is constructed on the antenna layer with a symmetric slot feeding. The grid element, as defined in [8] is implemented here. It is shown in Fig. 2. It consists of vertical microstrip lines with length $l_{\mathrm{g}}$, and width $w_{\mathrm{t}}$ and horizontal microstrip lines with length $w_{\mathrm{g}}$ and width $w_{\mathrm{a}}$. The length and width of the connecting lines between the grid elements are $c l_{1}$ and $c l_{\mathrm{w}}$ respectively. In the first simulation step, 2 grid elements are positioned, one on either side of the feeding slot as shown in Fig.2. Simulations are performed with 3 ports, with the grid antenna connected through microstrip

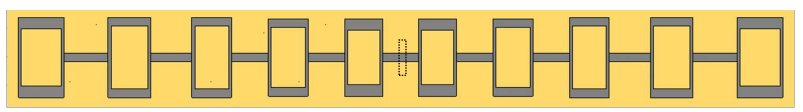

(a)

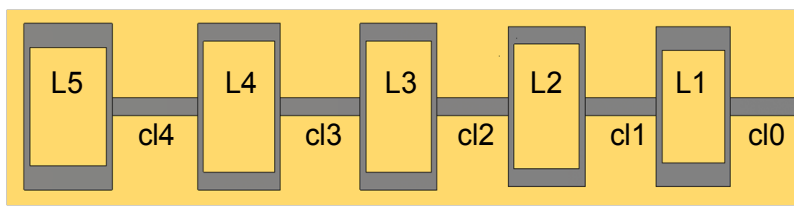

(b)

Figure 3: Top complete view (a) and left half view (b) of grid array antenna

TABLE I: Grid antenna array dimensions in $\mathrm{mm}$

\begin{tabular}{|c|c|c|c|c|}
\hline Rectangular loop & $l_{\mathrm{g}}$ & $w_{\mathrm{g}}$ & $w_{\mathrm{a}}$ & $w_{\mathrm{t}}$ \\
\hline L1 & 2.30 & 1.20 & 0.40 & 0.10 \\
\hline L2 & 2.40 & 1.25 & 0.30 & 0.10 \\
\hline L3 & 2.50 & 1.25 & 0.30 & 0.10 \\
\hline L4 & 2.50 & 1.35 & 0.30 & 0.10 \\
\hline L5 & 2.40 & 1.45 & 0.40 & 0.10 \\
\hline
\end{tabular}

TABLE II: SIW dimensions in $\mathrm{mm}$

\begin{tabular}{|c|c|c|c|c|c|}
\hline$a$ & $s l$ & $s d$ & $s w$ & $v d$ & $v p$ \\
\hline 2.00 & 1.00 & 0.95 & 0.3 & 0.30 & 0.60 \\
\hline
\end{tabular}

TABLE III: Dimensions of connecting lines in $\mathrm{mm}$

\begin{tabular}{|c|c|c|c|c|c|}
\hline Dimensions & $\mathrm{cl0}$ & $\mathrm{cl} 1$ & $\mathrm{cl} 2$ & $\mathrm{cl} 3$ & $\mathrm{cl} 4$ \\
\hline$c l_{1}$ & 1.20 & 1.25 & 1.30 & 1.40 & 1.50 \\
\hline$c l_{\mathrm{w}}$ & 0.30 & 0.30 & 0.30 & 0.30 & 0.30 \\
\hline
\end{tabular}

TABLE IV: Via type lengths and diameters in $\mathrm{mm}$

\begin{tabular}{|c|c|c|}
\hline Via type & Via length & Via diameter \\
\hline via $_{1}$ & 0.163 & 0.250 \\
\hline via $_{2}$ & 0.308 & 0.350 \\
\hline via $_{3}$ & 0.453 & 0.200 \\
\hline
\end{tabular}

lines to waveguide ports on both sides. The geometry of the slot as well as the grid elements are optimized for two simultaneous goals, i.e. a wideband impedance response and the required radiated power from the grid elements to achieve amplitude tapering over the array. In the next step, the number of grid elements is increased symmetrically by two and the optimization performed again. The process is repeated until there are 10 grid elements, at which point the power arriving at the output ports is negligible. At this point, the ports and the connecting microstrip lines to the ports are removed, and the array design is complete.

The final grid array antenna design is shown in Fig. 3. As mentioned earlier, the design is symmetric about the midpoint of $c l 0$. The antenna array dimensions, SIW feed dimensions and the dimensions of the connecting lines are listed in Tables I, II and III respectively.

\section{Microstrip to SIW transition}

The layer construction of the PCB is shown in Fig. 5. A wideband multilayer microstrip to SIW transition is designed to feed the SIW structure. The first part of the transition is a 


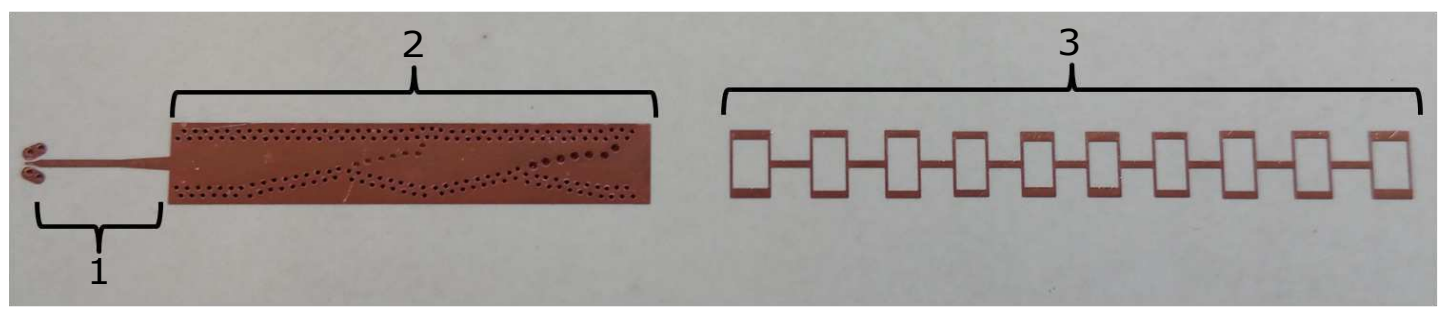

Figure 4: Top view PCB. 1: Microstrip line, 2: SIW transition, 3: Grid array antenna

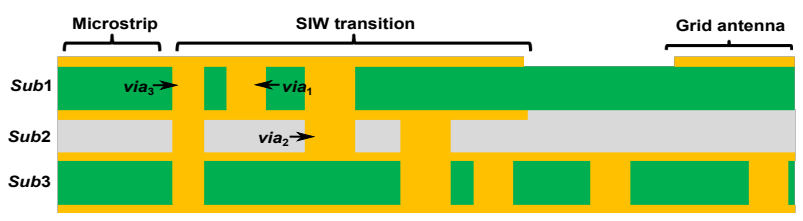

Figure 5: Layer construction of antenna PCB

tapered coplanar microstrip to SIW transition using the design equations in [10]. For a wideband mode transfer between substrate layers, the multilayer SIW transition in [11] is modified and implemented at $77 \mathrm{GHz}$ twice to transfer the mode first from $S u b 1$ to $S u b 2$ and then to $S u b 3$. The SIW in $S u b 3$ is extended to form the feeding SIW for the grid array antenna. To realize the transition in PCB technology, three different via types are used. The lengths and diameters for each via type are shown in Table IV. It can also be seen that the copper layer between $S u b 1$ and $S u b 2$ is only present in the region of the transition. This is designed so that the grid array antenna has a substrate thickness of $0.254 \mathrm{~mm}$ beneath it. The top view of the manufactured PCB is shown in Fig. 4.

\section{MeAsurement Results}

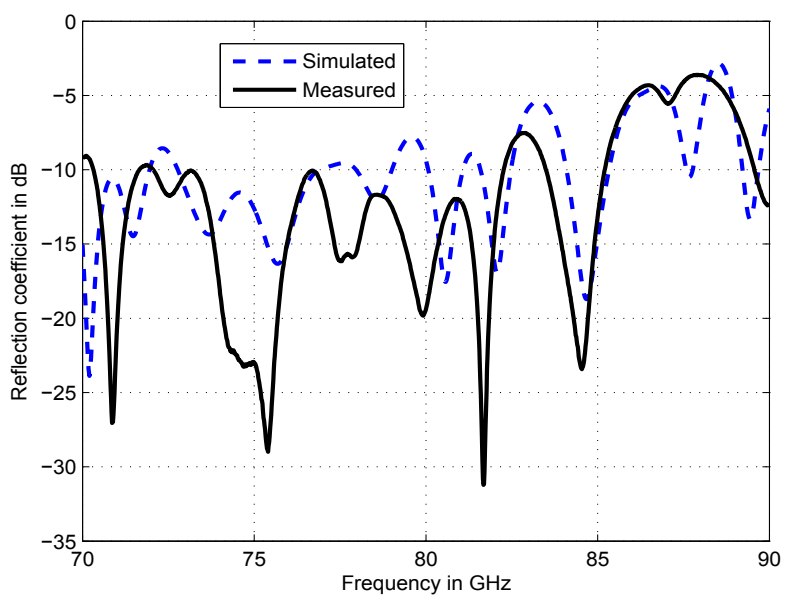

Figure 6: Reflection coefficient

The manufactured antenna was measured to verify the simulation results. Two antenna PCBs were manufactured. The first PCB employed a GSG-probe transition to verify the reflection coefficient. The second PCB employed the wideband waveguide transition from [12] with a $\mathrm{S} 11<-10 \mathrm{~dB}$ relative bandwidth of $15 \%$. This transition was used to measure radiation patterns. Reflection coefficient results are shown in Fig. 6. Wideband impedance matching was measured in the frequency range $73-82 \mathrm{GHz}$ (Reflection coefficient $<-10 \mathrm{~dB}$ ) for about $12 \%$ impedance bandwidth. The measured result is slightly shifted to higher frequencies compared to the simulation, which points to slightly lower actual dielectric constant values for the substrates than those used in the simulations.

Farfield radiation patterns for the E-Plane and H-Plane of the antenna are shown in Fig. 7 for the frequencies $77 \mathrm{GHz}$, $79 \mathrm{GHz}$ and $81 \mathrm{GHz}$. For the E-Plane results, it can be seen that when only the antenna is simulated, the farfield radiation pattern is smooth, the side-lobes levels are low and relatively symmetric with respect to the main lobe. However, when the complete structure including the designed microstrip to SIW transition, as well as the waveguide to microstrip transition is simulated, a high ripple, as well as an asymmetry in the radiation pattern is seen. This ripple and asymmetry are also observed in the measurements. Through simulations, the origin for the ripple has been determined to be the tapered microstrip to SIW junction. Parasitic radiation originating at this junction combines with the antenna radiation in the farfield and distorts the overall radiation pattern. A probable source of the asymmetry in the E-Plane radiation pattern is parasitic radiation at the waveguide to microstrip transition. Simulations show parasitic radiation in the direction Theta $\approx-50^{\circ}$ emanating at the microstrip output of this transition, which explains the higher side-lobes in the negative Theta direction.

In the H-Plane, a distortionless wide beam with $3 \mathrm{~dB}$ bandwidth of $48^{\circ}$ at $77 \mathrm{GHz}$ is achieved. In comparison to the simulations, the measured beamwidth is slightly less. The cause for this is the absorber material placed on the edges of the PCB which attenuates the radiated fields.

\section{CONCLUSION}

A microstrip grid array antenna, realized on a low profile RF substrate was presented. A novel feeding method, employing a transverse slot etched on the top metalization of an SIW, is used to feed the antenna array. The measured results of the SIW fed antenna show a wide impedance matching bandwidth, covering the operating frequency bandwidth of $76-81 \mathrm{GHz}$. A distorted and asymmetric radiation pattern is observed in the E-Plane simulation and measurement results. The cause for the distortion is the parasitic radiation at the junction of the tapered microstrip and SIW. This needs to be optimized to reduce the 


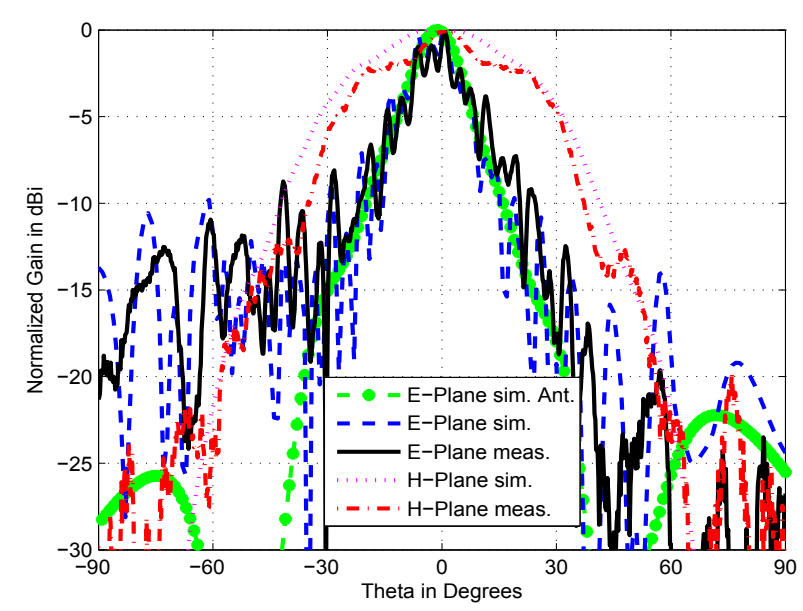

(a)

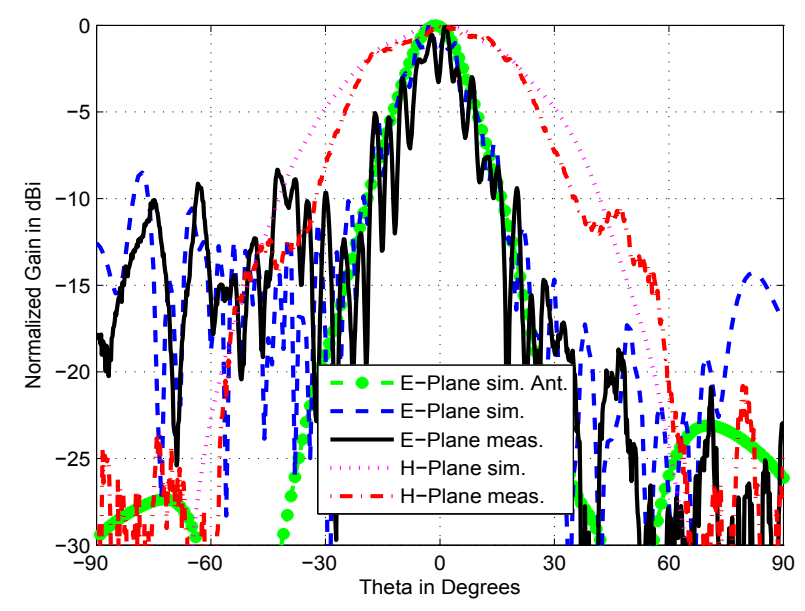

(b)

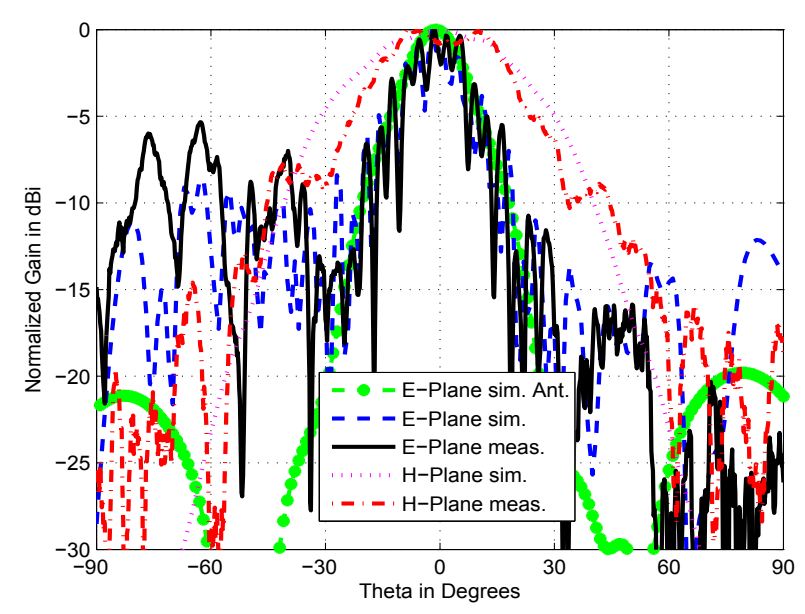

(c)

Figure 7: Normalized radiation pattern of antenna in E-Plane and H-Plane at (a) $77 \mathrm{GHz}$, (b) $79 \mathrm{GHz}$ and (c) $81 \mathrm{GHz}$ parasitic radiation. A probable cause of the asymmetry is the parasitic radiation at the output of the waveguide to microstrip transition. Back-fed transitions, such as in [13] can be used to overcome this parasitic radiation and improve the measured antenna performance.

\section{REFERENCES}

[1] European Commission: Commission Impelementing Decision 2011/485/EU of 29 July 2011 amending Decision 2005/50/EC on the harmonisation of the $24 \mathrm{GHz}$ range radio spectrum band for the time-limited use by automotive short-range radar equipment in the Community (notified under document C(2011) 5444). Official Journal of the European Union L 198/71, (2011).

[2] W. Menzel and A. Moebius, "Antenna concepts for millimeter-wave automotive radar sensors," Proceedings of the IEEE, vol. 100, no. 7, pp. 2372-2379, July 2012

[3] J. Kraus, "A backward angle-fire array antenna," IEEE Transactions on Antennas and Propagation, vol. 12, no. 1, pp. 48-50, 1964.

[4] R. Conti, J. Toth, T. Dowling, and J. Weiss, "The wire grid microstrip antenna," IEEE Transactions on Antennas and Propagation, vol. 29 no. 1, pp. 157-166, 1981.

[5] B. Zhang and Y. P. Zhang, "Grid array antennas with subarrays and multiple feeds for 60-GHz radios," IEEE Transactions on Antennas and Propagation, vol. 60, no. 5, pp. 2270-2275, 2012.

[6] F. Bauer and W. Menzel, "A $79 \mathrm{GHz}$ microstrip grid array antenna using a laminated waveguide feed in LTCC," in IEEE International Symposium on Antennas and Propagation (APSURSI), 2011, pp. 2067-2070.

[7] M. Frei, F. Bauer, W. Menzel, A. Stelzer, and L. Maurer, "A $79 \mathrm{GHz}$ differentially fed grid array antenna," in European Radar Conference (EuRAD), Oct 2011, pp. 432-435.

[8] O. Khan, J. Pontes, X. Li, and C. Waldschmidt, "A wideband variable width microstrip grid array antenna," in 2014 European Radar Conference (EuRAD), Oct 2014, pp. 341-344.

[9] W. Abdel-Wahab and S. Safavi-Naeini, "Wide-bandwidth 60-ghz aperture-coupled microstrip patch antennas (mpas) fed by substrate integrated waveguide (siw)," IEEE Antennas and Wireless Propagation Letters, vol. 10, pp. 1003-1005, 2011.

[10] D. Deslandes, "Design equations for tapered microstrip-to-substrate integrated waveguide transitions," in IEEE MTT-S International Microwave Symposium Digest (MTT), May 2010, pp. 704-707.

[11] H. Abuzaid, A. Doghri, K. Wu, and A. Shamim, "Siw based multilayer transition and power divider in ltcc technology," in IEEE MTT-S International Microwave Symposium Digest (IMS), June 2013, pp. 1-3.

[12] E. Topak, J. Hasch, and T. Zwick, "Compact topside millimeter-wave waveguide-to-microstrip transitions," IEEE Microwave and Wireless Components Letters, vol. 23, no. 12, pp. 641-643, Dec 2013.

[13] K. Sakakibara, M. Hirono, N. Kikuma, and H. Hirayama, "Broadband and planar microstrip-to-waveguide transitions in millimeter-wave band," in International Conference on Microwave and Millimeter Wave Technology (ICMMT), vol. 3, April 2008, pp. 1278-1281. 